\title{
Covid-19: gastroenterology perspectives
}

\begin{abstract}
Natassia Vianna Bocchese'; Noele Gurgel Dávila ${ }^{1}$; Maria Beatriz Maia Rodrigues'; Rebecca Renata Lapenda do Monte ${ }^{1}$; Renata Carneiro

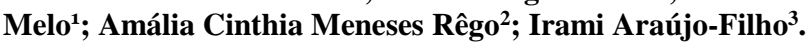

${ }^{1}$ Undergraduate Student of Medicine at UnP - Potiguar University - Laureate International Universities - Natal/Brazil;

${ }^{2}$ Postgraduate Program in Biotechnology at Potiguar University / UnP - Laureate International Universities. Teaching and Research Manager - School of Health - League against Cancer Natal - RN / Brazil. Ph.D. in Health Science;

${ }^{3}$ Postgraduate Program in Biotechnology at Potiguar University/ UnP-Laureate nternational Universities; Full Professor Department of Surgery, Federal University of Rio Grande do Norte. Full Professor, Department of Surgery, Potiguar University. Ph.D in Health Science/ Natal-RN, Brazil.
\end{abstract}

*Corresponding Author: Irami Araújo-Neto, Undergraduate Student of Medicine at UnP - Potiguar University - Laureate International Universities - Natal/Brazil.

\section{Received date: May 26 2020; Accepted date: June 15, 2020; Published date: June 22, 2020}

Citation: Natassia Vianna Bocchese, Noele Gurgel Dávila, Maria Beatriz Maia Rodrigues, Irami Araújo-Neto, Mariana de Oliveira Costa, Diniz de Meiroz Grillo Barbalho, Amália Cinthia Meneses Rêgo, Irami Araújo-Filho. Clinical Medical Reviews and Reports. 2(3); DOI: 10.31579/2690$8794 / 021$

Copyright: () 2020 Natassia Vianna Bocchese, This is an open-access article distributed under the terms of the Creative Commons Attribution License, which permits unrestricted use, distribution, and reproduction in any medium, provided the original author and source are credited

\section{Abstract}

The clinical syndrome caused by SARS-CoV-2 was called COVID-19 and became a pandemic from March 2020. Fever, dry cough, and dyspnoea are the predominant clinical manifestations of the disease. However, symptoms such as diarrhea, nausea, vomiting, and abdominal discomfort can also be found in infected patients. Despite the increased prevalence since the second phase of the recent epidemic in China, scientific evidence on the gastrointestinal manifestations of COVID-19 has received little attention. This study aimed to report gastrointestinal signs and pathological findings in patients with COVID-19, in addition to discussing the possibility of fecal transmission. SARS-CoV-2 penetrates gastrointestinal epithelial cells, making patients' feces potentially infectious. Although viral RNA can be detected in feces, it is not clear whether fecal-oral transmission occurs. The gastrointestinal characteristics and abdominal manifestations of COVID-19 were reviewed based on case reports and retrospective clinical studies related to the digestive system. It is essential to know that, as the pandemic spreads, new studies are published, and knowledge about gastrointestinal symptoms is better known and reported.

Keywords: COVID-19, COVID-19 virus disease, SARS-CoV-2 infection, Signs and Symptoms - Digestive, Gastrointestinal Tract, Digestive System.

\section{Introduction}

The SARS-CoV-2 virus, the causative agent of COVID-19, has been detected in more than 166 countries worldwide. The disease began in China in 2019. Due to its rapid spread, the World Health Organization (WHO) declared COVID-19 as a pandemic. This situation has affected several countries and has become a threat to public health and a socioeconomic challenge [1-5].

Coronaviruses belong to a family of single-stranded RNA viruses, which are subdivided into four main genera. The taxonomy of SARS-CoV-2 is $82 \%$ similar to that of the SARS-CoV-1 coronavirus, and both belong to the $\beta$ genus. The clinical manifestations of these diseases are similar and involve the respiratory and digestive systems [6-9].

Although the symptoms of SARS-CoV-2 are milder, infectivity is higher compared to SARS-CoV and MERS-CoV, becoming a health threat. Generally, the clinical syndrome of COVID-19 is associated with fever, dry cough, and dyspnoea. However, atypical symptoms such as weakness, myalgia, diarrhea, nausea, vomiting, and neurological conditions are described in some patients. Gastrointestinal symptoms, although rare, it is possible and harder to recognize as part of the usual syndrome $[1,7,8,10,11]$.
In addition to the usual clinical picture, there is the possibility that infected patients may be asymptomatic, with a more aggressive lateonset spectrum of disease, severe acute respiratory failure, and multiple organ failure, due to increased inflammatory cytokines. These changes increase the morbidity and mortality of the disease $[1,2,8,10]$.

It was observed that during the initial stage of the epidemic, the proportion of patients with diarrhea and other digestive symptoms reported both in Wuhan and in different regions was around $2 \%-10 \%$. However, in the late phase of the epidemic, the number of these patients increased significantly [1].

To better understand the involvement of the gastrointestinal system, the present study analyzed published clinical cases with reports of digestive manifestations and pathological findings of patients with COVID-19 to provide references for prevention and control, diagnosis, and treatment of this new pathology when its clinical presentation differs from the usual.

\section{Methods}

The research was carried out in the databases Pubmed, Scopus, Scielo, Embase, Web of Science, in addition to searching Google Scholar, considered as a source of gray literature, as it does not contain peer- 
reviewed articles. The articles were selected according to the focus of the study: manifestations of the gastrointestinal and abdominal tracts of COVID-19, in addition to research of the fecal-oral transmission. The studies were chosen by combining the following keywords: COVID-19, COVID-19 virus disease, SARS-CoV-2 infection, Signs, and Symptoms Digestive, Gastrointestinal Tract, Digestive System. All relevant studies published in the 2019-2020 period were included in the cohort, systematic review, case-control, cross-sectional, case series, review and editorial studies. The filters chosen were studies carried out in humans, complete and published from January 2019 to the present, totaling thirty articles. The analysis, review, and selection of materials was carried out in pairs, separately, based on the reading of the title and summary of the study, with a third reviewer in case of disagreement between the other two reviewers.

\section{Results and Discussion}

Based on the similarity with the other viruses of the coronavirus family, some studies question the possibility of fecal-oral transmission of SARS-CoV-2, in addition to transfer by droplets, aerosols, and direct contact. The data are emphatic when they state that the SARS-CoV-2 and MERS-CoV-2 viruses are viable in contaminated water and feces, being considered potential means of transmission [7].

Years ago, the RNA of these viruses was isolated in the sewage of a hospital in Beijing that treated patients with SARS, and another study was carried out, ratifying the growth of SARS-CoV-1 in the sewage water. Other hospitals through these studies, it was also proven that the viruses remain highly infectious for 14 days, being able to withstand temperatures of up to $4^{\circ} \mathrm{C}$.

However, despite the structural similarity of these viruses with SARS-CoV-2, there is still a consensus on the resistance and variability. Yet, it is known that fecal samples remain positive for 11.2 days, on average, after clearance of respiratory tract samples $[7,12]$

According to Yeo et al., the viability of SARS-CoV-1 and MERS-CoV is proven in conditions of low temperatures and humidity, as well as the continued presence of these agents in the environment, corroborating their transmissibility by contact contaminated fomites. Thus, it was observed that these viruses present tropism for the cells of the gastrointestinal tract, and their presence remains viable in the patients' feces, even after hospital discharge. These findings provide us with explanations about the occurrence of gastrointestinal symptoms, the recurrence in patients considered cured, and the transmission in close contacts $[7,13]$.

In any case, the fecal-oral transmission is more common in rural regions where there is no primary sanitation network and adequate sanitary facilities. Besides, the population lacks awareness of the need for hand hygiene, as well as the habit of drinking contaminated water and food [14].

The epidemiological profile of patients affected by COVID-19 varies according to the population studied. Still, middle-aged women, smokers, and patients with heart disease, diabetes, or chronic obstructive pulmonary disease are more affected in general. In addition to typical respiratory symptoms, about $19 \%$ of these patients had at least one gastrointestinal manifestation at some point, with diarrhea being the most common $[14,15]$.

Enteric disease with mild and nonspecific characteristics, when not associated with respiratory impairment, was neglected and underestimated during the first clinical investigations of COVID-19, corroborating the critical state that the pandemic is today $[15,16]$.
Therefore, health professionals should identify these patients early and warn them about the possible risk of infection after confirmation through the oropharyngeal swab [13].

In patients with pre-existing digestive diseases, clinical outcomes are often more reserved. In a political analysis in China involving 1590 infected with COVID-19, there were 18 cases $(1 \%)$ of previous cancer, 3 of which were related to colorectal cancer [12,13].

Patients with COVID-19 and cancer had a higher risk of severe events because the expression of angiotensin-converting enzyme receptor 2 (ACE2) is higher in the colon epithelial cells of patients with adenoma or colorectal cancer. Such an association predisposes a higher risk of infection by COVID-19 $(12,14)$.

Patients with inflammatory bowel disease (IBD) have a worse risk of developing a severe infection or disease course since they have continued involvement and use corticosteroids and other immunosuppressive agentes $[15,16]$.

\section{Pathophysiological changes}

The human coronavirus has transmissibility and pathogenicity associated with recognition and binding to receptors. Protease cleavage and membrane fusion are resembling about $73 \%$ with SARS-CoV-1 [14$16]$.

One of the receptors involved in this process is related to the angiotensin-converting enzyme 2 (ACE2), which is expressed in pulmonary type 2 macrophages, as well as in squamous esophageal cells and ileum and colon enterocytes [13].

Therefore, several pathological findings of the gastrointestinal system were observed in patients with COVID-19. Intestinal segmental dilation or stenosis, degeneration, necrosis, and edema of the gastrointestinal mucosa in histology $[1,6]$.

Despite this, Tian et al. did not report epithelial destruction of the esophagus, stomach, duodenum, and colorectal mucosa through hematoxylin and eosin (HE) staining. Histology only indicated lymphocytic infiltration in the esophageal squamous epithelium, in addition to abundant plasma cells and lymphocytes infiltrated with interstitial edema in the stomach, duodenum and lamina propria of the rectum [1].

By observing the ACE2 cell receptor and viral nucleocapsid protein in tissue samples, it was shown that the positive areas were mainly distributed in the cytoplasm of the epithelial cells and the cilia of the gastrointestinal glandular epithelial cells [9-11].

Such changes were not found in the esophageal squamous epithelial cells. Similar receptors were observed in the stomach, duodenum, and rectal epithelial cells, suggesting that ACE2 receptors facilitate the entry of the virus into the gastrointestinal tract (GIT) $[1,17]$

The SARS-CoV-2 use ACE2 as a receptor on the host does it more efficiently than the strain identified in SARS-CoV-1 in 2003 [7]. However, even though the ACE2 receptor is found in TGI cells, more abundantly in patients positive for COVID-19 [7-9].

It cannot be concluded with certainty whether such an enzyme is decisive for the entry of the virus into the cell, and consequently is responsible for pathogenesis since higher levels of this enzyme were also found in the kidney of affected patients. Only a small portion of them was found viral RNA in the urine $[17,18]$.

It is suggested that the virus can bind to cholangiocytes via the ACE2 receptor. This was confirmed by the presence of microvesicular 
steatosis and mild lobular activity in liver biopsy in the post-death of a patient with COVID-19. These changes suggest direct involvement by the virus or liver damage caused by drugs. However, no viral inclusion was seen in the liver [19].

Besides, there was the presence of mild to moderate liver damage associated with increased aminotransferases, hypoproteinemia and prolonged prothrombin time. It is worth noting that $60 \%$ of patients affected with SARS are suffering from liver failure, so care should be taken about these disorders in patients with COVID-19 [19-21].

SARS-associated hepatotoxicity is due to viral hepatitis or a side effect associated with the toxicity of antiviral drugs used in high doses. Despite the similarity between the two viruses, little is known about liver involvement in SARS-CoV-2 infection, but there is evidence of different degrees of liver dysfunction $[12,13]$.

TGI manifestations, in general, can occur through three main mechanisms. The first occurs through the direct attack of the virus through a chain of inflammatory responses, which increases the permeability of the TGI wall and hinders absorption by enterocytes, culminating in diarrhea. It is corroborated by the detection of viral nucleocapsid protein in epithelial cells [15-17].

The second occurs through the use of antiviral drugs used to treat these patients since they induce nausea and diarrhea. The third occurs due to changes in the intestinal microbiota caused by the use of antibiotics, precipitating digestive symptoms by an indirect mechanism $[1,13]$.

\section{Syndromic aspects}

The set of signs and symptoms caused by COVID-19 ranges from mild respiratory tract infection to severe pneumonia, acute respiratory distress syndrome, and multiple organ dysfunction. Fever associated with respiratory symptoms is more common, and the scientific community is still discovering extrapulmonary manifestations [12].

According to a cross-sectional study that included 204 patients with COVID-19 admitted to 3 hospitals in Hubei province, 99 patients (43.5\%) had symptoms of GIT as the chief complaint, and 7 of these patients did not have any respiratory symptoms [12]

Thus, despite being non-specific symptoms of the disease, retrospective studies have already proved that intestinal involvement occurs in a real way. Tian et al. highlighted anorexia as the most common gastrointestinal symptom, followed by diarrhea, vomiting, nausea, and abdominal pain. Anorexia results from the inflammatory process, hypoxia, damage to liver function, depression, or side effects of medications $[1,12]$

Corroborating this finding, Musa and Wong et al. found that $48.5 \%$ of the patients had gastrointestinal symptoms, $20 \%$ of whom had diarrhea, vomiting, or abdominal pain. Although diarrhea is one of the most prevalent gastrointestinal symptoms, the presence of constipation has been proven. It was seen that mild symptoms usually last 3.7 days on average, and most have a self-limiting evolution. $(1,12,19)$

Zhang et al. studying the clinical characteristics of 140 patients in Wuhan, he found that more than a third of them had abdominal pain, nausea, vomiting, diarrhea, and anorexia $[18,20]$.

Even after the improvement in the initial clinical picture, COVID-19-induced diarrhea persisted. The intestinal damage by the coronavirus promotes malabsorption in other symptoms of the disease [18].
Patients without digestive symptoms are more likely to be cured and discharged when compared to those with enteric involvement. This phenomenon is due to the intense viral replication in the GIT, leading to more severe disease, or too late diagnosis, in cases where there is no associated respiratory impairment [12].

Given the above, it is necessary to pay attention to cases that present only with manifestations of TGI. Cheung KS et al. demonstrated in a meta-analysis that part of the patients did not report respiratory symptoms or fever, but presented gastrointestinal symptoms, including anorexia, in $66.7 \%$ of the cases [17].

Studies corroborate the fact that critically ill patients are more likely to manifest digestive changes. It is believed that the high rate of severe cases indicates a more significant amount of virus and infectivity. Abdominal pain was more present in patients admitted to the intensive care unit than in those stable. The exact reason for explaining this has not yet been elucidated, but it should be discovered in future clinical trials with more sound samples $(1,17)$.

Henry BM et al. observed that abdominal pain is associated with four times higher risk of developing critical illness in patients with nausea and vomiting; however, in contrast, there was no association with diarrhea [21].

It is worth noting that long-term hypoxemia and cell necrosis caused by Watery Respiratory Discomfort Syndrome (SRDA) causes damage to the gastrointestinal mucosa, ulceration, and bleeding. The use of corticosteroids, anti-inflammatories, and the metabolic response to stress affect the GIT mucosa. Therefore, analyzing whether gastrointestinal signs and symptoms are causes or consequences of the clinical condition is severe $[1,8]$

Other extrapulmonary manifestations of COVID-19 are the acute abdomen. A 53-year-old patient initially presented with symptoms of bloating, malaise, and fever. During the directed anamnesis, he referred to respiratory symptoms, such as a flu-like condition; but he denied dyspnea, arthralgia, and myalgia. During the evolution of the disease, the patient had distended abdominal loop suggestive of infectious colitis and a positive test for SARS-CoV-2. (6)

This case is essential to show the importance of directed anamnesis and the active search for patients with respiratory symptoms, even if at the time it appears to be a surgical or abdominal pathology. The prompt identification of these patients allows for isolation and immediate intervention. Therefore, we must investigate the presence of cough and fever independently. (4-6)

We don't have a report of visceral perforation, mesenteric ischemia, or gastrointestinal bleeding in adults. However, there have been cases of hematochezia in children and neonates in critical condition. It is worth noting that rectal bleeding is not common in COVID-19, and should serve as a warning for other differential diagnoses $[6,14,16]$.

Furthermore, in this moment of instability that society is experiencing, we should not underestimate the negative emotions that can simulate symptoms of IBD and irritable bowel syndrome [14].

A new fact presented by the researchers is the association of metabolic disorders with the dysfunction of the central nervous system and intestine axis. Some studies reveal that there are changes in the intestinal microbiota and an increase in the absorption of harmful metabolites due to the overexpression of enteric ACE2 receptors, which can cause dizziness, syncope, and fatigue [20-22].

In this regard, although gastrointestinal symptoms are less common in patients with COVID-19, the diagnosis cannot be excluded 
and should be investigated in all patients referred to the Emergency Department. A viable and fast alternative is the use of lung ultrasound at the bedside to detect signs of interstitial pneumonia, even in the absence of respiratory symptoms, as it helps in the early detection of other manifestations, in addition to those of GIT [11 -13].

\section{Liver damage}

The Liver damage may also be present in patients affected by COVID-19. The cytopathic effects induced by the virus cause direct hepatotoxicity. There is consistent evidence that SARS-CoV-2 binds directly to the cholangiocyte ACE2 receptor and disrupts liver function. Thus, gamma-glutamyltransferase $\%$ of the patients analyzed, while only $1.8 \%$ had a high level of alkaline phosphatase (AF) in recent trials $[12,23]$.

Another explanation for this involvement is the high levels of positive end-expiratory pressure in patients using mechanical ventilation, since it can increase hepatic congestion, due to the increase in right blood pressure and consequent difficulty in venous return [8-10]. It is important to note that drug-induced liver injury should be investigated. The most associated drugs are Lopinavir and Ritonavir, in addition to acetaminophen and antibiotics [12,23-25].

In patients with previous liver disease, the clinical course is more unfavorable, with the development of severe hepatitis due to increased viral replication during infection $[11,12]$. On the other hand, patients with hepatitis B and COVID-19 were observed in China, who did not have hepatic repercussions of greater severity, when compared to those without previous pathology [23]

\section{Pediatric patients}

In children, more cases of vomiting were observed when compared to adults, and up to $10 \%$ of children had only gastrointestinal symptoms, instead of respiratory symptoms at the beginning of the disease. The hypothesis formulated is that children should be more prone to gastrointestinal symptoms than respiratory symptoms. Another possibility is due to the immature immune system of children that can reduce the immune response in the respiratory system. However, no conclusions can be drawn, as there is a small number of cases in patients under 12 years of age [1-3].

\section{Complementary exams}

Due to the similar taxonomy of SARS-Cov-1 and MERS-CoV with SARS-CoV-2, it is common to associate their syndromic and laboratory aspects. Previous studies have shown that SARS-CoV RNA was detected in feces only from the fifth day of the disease. Besides, the proportion of fecal samples positive for viral RNA increased progressively, reaching maximum concentration on the eleventh day of infection and was present in the stool of a small portion of patients even after 30 days $[7,17,24]$

Tan Y. et al. observed in a retrospective study that even 15 days after the beginning of the clinical manifestations of COVID-19, viral particles of SARS-Cov-2 are still detected in fecal samples, contributing to the hypothesis that even after the elimination of the upper respiratory tract virus, it is still present in the gastrointestinal tract [25]. Not only were rectal swab and fecal samples positive, but viruses were also detected in saliva, urine, esophageal erosion, and bleeding from severe peptic ulcers $[17,24]$.

In addition to the respiratory and gastrointestinal tracts, the virus is in the blood of infected individuals. Therefore, a confirmed case is considered to be one with a compatible clinic associated with the presence of viral RNA in the blood or other culture medium. The presence of the virus alone in the feces has not yet been considered a standard for making the diagnosis of the disease [10,17,24-26].

For Chen et al., the presence of viral RNA in the feces did not correlate with the severity of respiratory symptoms and gastrointestinal manifestations [24].

Thus, the first step in investigating patients with suspected COVID-19 with only gastrointestinal manifestations is to look for other suggestive symptoms. The patient usually progresses with lymphocytopenia, thrombocytopenia, detection of SARS-CoV-2 nucleic acid in the nasopharyngeal swab, and presence of a ground-glass lesion on chest tomography. These tests are essential to differentiate the gastrointestinal symptoms of COVID-19 from other differential diagnoses [16].

As previously mentioned, research has shown liver changes of varying degrees. The elevation of liver enzyme levels with ALT ranged between $16 \%-53 \%$. Another study found an increase in total bilirubin, AST, and ALT in $10 \%, 21 \%$, and $22 \%$ of patients, respectively. Changes in GGT and AF, too, may suggest liver involvement [18,23,27].

According to Wang et al., There was an increase in fecal calprotectin (CF) in those patients infected with COVID-19 who developed diarrheal conditions. Forty patients were evaluated, 55\% of whom reported diarrhea. The concentration of CF was closely related to the serum concentration of interleukin-6, thus demonstrating a link with a critical intestinal inflammatory response [28].

The decision to discharge patients infected with SARS-CoV-2 should be based on the negative results of at least two samples of the respiratory tract collected within 24 hours, in addition to isolation for 14 days after the high [27,28].

Despite these recommendations, it is crucial to guide routine hand hygiene, cleaning bathrooms, and avoid sharing this environment with other family members. Because of what was previously seen concerning the presence of the virus in the feces, it has been suggested to add the RT-PCR analysis of fecal samples as part criteria hospital discharge $[1,12,26]$.

\section{Gastrointestinal Surgery}

There is no denying the need for surgical treatment in most abdominal conditions. There are numerous therapeutic options, and the number of comorbidities that require surgical interventions is growing. However, in the face of this pandemic, all efforts should be aimed at reducing the risk of contamination in high-risk patients, including surgical ones. Thus, surgeons must make accurate diagnoses and need to weigh the risks and benefits involved in operative treatments $[10,13]$.

Abdominal surgeries have a higher risk of perioperative respiratory complications. All respiratory dynamics are modified, either by the use of assisted ventilation (orotracheal intubation) or by the surgical procedure itself, which can lead to an increase in intra-abdominal pressure, postoperative pain, and cavity bleeding [7,10,21]

The laparoscopic technique involves artificial pneumoperitoneum, performed with the use of carbon dioxide $(\mathrm{CO} 2)$. This process increases intra-abdominal pressure and retains pulmonary $\mathrm{CO} 2$ and reduces gas Exchange [8-10].

In the critical surgical patient, the release of inflammatory cytokines is a risk factor for severe COVID-19 infection. Therefore, all efforts to avoid unnecessary surgeries and, especially, outside the context of urgency and emergency, must be instituted by the team surgeon to reduce the morbidity and mortality associated with COVID infection 19 
as well as the contamination of the surgical team, in cases of infected but asymptomatic patients undergoing elective surgery [10,24-26].

However, inevitably, during the pandemic, there will be situations in which urgent surgery will be necessary. During the SARS outbreak, the transmission of infection from the donor to the recipient was reported during liver transplant surgery. Therefore, all precautions should be taken to reduce the risks of disease transmission [13]

\section{Immune suppression}

Patients with gastrointestinal diseases, inflammatory bowel disease (IBD), usually use infliximab, 5-aminosalicylic acid, thalidomide, among others. Given the immunosuppression that these drugs can cause, the Chinese Society of Gastroenterology temporarily canceled the use of these classes of drugs, to avoid worse affections by COVID-19 [11-13].

In patients with IBD, hospitals have launched new therapeutic strategies for their patients, as the number of IBD-contaminated individuals was growing, with cases being reported in France, Italy, Spain, and the USA $[15,28]$

People with IBD are susceptible to frequent and severe infections. To stop this contamination, risk assessment, stricter prevention measures, patient education, contact by phone or internet, containment of patients with the same pathology in specific rooms, and delivery of medicines to those who lived in places were adopted distant $[4,12,15]$.

These strategies were essential and contributed to the end of confirmed cases in the hospital in China. Such results proved that mass education is vital, and the implementation of stricter measures for immunosuppressed patients is crucial $(15,19-21)$

Despite the recommendation being the total suspension of immunosuppression in patients, An P. et al. believe that this action should be taken according to the location addressed and the incidence rate of COVID-19 in this region [15,17-19].

\section{Endoscopy}

Contagion prevention measures, mainly by health professionals, are valid and reduce the chance of infection for these workers as well as patients. It is known that there is a higher risk of contagion through droplets in contact with mucous membranes and conjunctivae. For this reason, several entities and specialty societies assist in this regard $[21,26]$.

However, it is estimated that about $10 \%$ of healthcare professionals become infected with the virus. The use of personal protective equipment, correct risk stratification of patients and isolation of suspected cases are measures that can reduce the risk of contagion $[3,9,22]$.

In the context of the pandemic we are experiencing, surgical procedures and more invasive exams should only be performed in cases of extreme urgency and need. In endoscopy units, there is a high risk of droplet inhalation, conjunctival contact, and contamination during the examination. Coughing, choking, and vomiting may occur during endoscopy, just as flatus and liquid stools containing pathogens may be present during colonoscopy [16,20-22].

For this reason, the number of invasive tests performed during the COVID-19 outbreak should be limited, is indicated only in cases of digestive bleeding, presence of foreign bodies in the GIT, acute cholangitis secondary to bile duct obstruction and in situations that represent a risk of death. However, before the exam is performed, it is necessary to assess the patient for the presence of fever, respiratory, and gastrointestinal symptoms, as well as the laboratory diagnosis through the swab [16, 28-30].

Through the clinical approach, RT-PCR test for SARS-CoV-2 on nasopharyngeal swab and chest computed tomography; patients should be classified as low, moderate, and high risk of transmission. Thus, the risks and benefits related to each patient should be considered for the examination. It is worth mentioning that the slow performance of diagnostic colonoscopy can increase the risk of high-grade dysplasia and colorectal câncer [5-7,16]

The procedures must be carried out in an isolated room with negative pressure; however, when this is not possible, ventilation must be adequate. Endoscopic accessories must be sterilized appropriately or use disposable accessories. Besides, professionals must be dressed in personal protective equipment (PPE), goggles, hat, mask, along with resistant clothing and gloves $[10,29]$

For Tao et al., the products used for disinfection are ether, $75 \%$ alcohol, fatty solvents, temperatures above $56^{\circ} \mathrm{C}$, disinfectants with chlorine in their composition, and peroxyacetic and chloroform acids Also, ultraviolet radiation and ozone must be used in endoscopy units to clean and sterilize the environment $[10,21,26]$.

If they are not available, it is necessary to open the windows of the endoscopy room and wait for about an hour between procedures. All these measures must be taken carefully, since it has been proven, through an environmental study, that SARS-CoV-2 can remain viable in aerosols and stable in plastic and stainless steel for more than $72 \mathrm{~h}[10,16,19,30]$.

\section{Conclusion}

The pandemic caused by the SARS-CoV-2 virus has become a public health problem, in addition to generating a social and economic crisis in several countries around the world. Several studies are underway to clarify the clinical manifestations caused by the infection and to clarify the forms of transmission.

However, it is known that, in addition to respiratory symptoms, patients can start COVID-19 with the impaired gastrointestinal tract. Therefore, suspicion of COVID-19 should be raised, especially in highrisk patients, when there are digestive symptoms, even in the absence of respiratory symptoms. It is essential for health professionals to be aware of the extrapulmonary involvement of the disease, as they will help in the early identification of COVID-19.

All information about these atypical manifestations can have a significant impact on inhibiting transmission, especially in hospitalized patients, as well as on early treatment and better prognosis. Also, it was observed that the disease could be transmitted via the fecal-oral route, and therefore health measures must be taken effectively.

Depending on the World Health Organization (WHO), we believe that early suspicion and diagnosis are essential to contain the global spread of COVID-19 infection and preventive measures are crucial to stop the spread of the virus and prevent devastating outbreaks of the disease.

\section{Compliance with ethical standards}

\section{Acknowledgments}

The authors thank the Ph.D. in Health Sciences and Teaching and Research Manager at League Against Cancer, Profa. Dra. Amália Rêgo, for her contribution and relevance to the scientific discussion and supervision of this research, acting as an expert consultant on the bibliographic survey, analysis, and scientific advice. We also thank all the 
study components for their dedication and effort to build a scientifically validated quality study.

\section{Disclosure of conflict of interest}

There are no conflicts of interest to declare by any of the authors of this study.

\section{References}

1. Tian Y, Rong L, Nian W, He Y. Review article: gastrointestinal features in COVID-19 and the possibility of faecal transmission. Aliment Pharmacol Ther. 2020;51(9):843-851.

2. Gralnek IM, Hassan C, Beilenhoff U, Antonelli G, Ebigbo A, Pellisè M, Arvanitakis M, Bhandari P, Bisschops R, Van Hooft JE, Kaminski MF, Triantafyllou K, Webster G, Pohl H, Dunkley I, Fehrke B, Gazic M, Gjergek T, Maasen S, Waagenes W, de Pater M, Ponchon T, Siersema PD, Messmann H, DinisRibeiro M. ESGE and ESGENA Position Statement on gastrointestinal endoscopy and the COVID-19 pandemic. Endoscopy. 2020;17.

3. Singhal T. A Review of Coronavirus Disease-2019 (COVID19). Indian J Pediatr. 2020;87(4):281-286.

4. Kannan S, Shaik Syed Ali P, Sheeza A, Hemalatha K. COVID19 (Novel Coronavirus 2019) - recent trends. Eur Rev Med Pharmacol Sci. 2020;24(4):2006-2011.

5. Ozma MA, Maroufi P, Khodadadi E, Köse Ş, Esposito I, Ganbarov K, Dao S, Esposito S, Dal T, Zeinalzadeh E, Kafil HS. Clinical manifestation, diagnosis, prevention and control of SARS-CoV-2 (COVID-19) during the outbreak period. Infez Med. 2020; 1;28(2):153-165.

6. Blanco-Colino R, Vilallonga R, Martín R, Petrola C, Armengol M. Suspected acute abdomen as an extrapulmonary manifestation of Covid-19 infection. Cir Esp. 2020;98(5):295296.

7. Yeo C, Kaushal S, Yeo D. Enteric involvement of coronaviruses: is faecal-oral transmission of SARS-CoV-2 possible? Lancet Gastroenterol Hepatol. 2020.5(4):335-337.

8. Zhu J, Ji P, Pang J, Zhong Z, Li H, He C, Zhang J, Zhao C. Clinical characteristics of 3,062 COVID-19 patients: a metaanalysis. J Med Virol. 2020.15.

9. Ramanathan K, Antognini D, Combes A, Paden M, Zakhary B, Ogino M, et al. Since January 2020 Elsevier has created a COVID-19 resource centre with free information in English and Mandarin on the novel coronavirus COVID- research that is available on the COVID-19 resource centre - including this for unrestricted research re-use a. 2020;(January):19-21.

10. Tao KX, Zhang BX, Zhang P, Zhu P, Wang GB, Chen XP; General Surgery Branch of Hubei Medical Association; General Surgery Branch of Wuhan Medical Association. Recommendations for general surgery clinical practice in novel coronavírus pneumonia situation. Zhonghua Wai Ke Za Zhi. 2020;14;58(0):E001.

11. Ribeiro F, Bibi M, Pereira M, Ferreira S, Pessegueiro H, Araújo R. Severe Acute Liver Injury Related to Heat Stroke. Eur J Case Rep Intern Med. 2020;1;7(2):001382.

12. Musa S. Hepatic and gastrointestinal involvement in coronavirus disease 2019 (COVID-19): What do we know till now? Arab J Gastroenterol. 2020;21(1):3-8.

13. $\mathrm{Gu}$ J, Han B, Wang J. COVID-19: Gastrointestinal Manifestations and Potential Fecal-Oral Transmission. Gastroenterology. 2020;158(6):1518-1519.

14. Li LY, Wu W, Chen S, Gu JW, Li XL, Song HJ, Du F, Wang G, Zhong CQ, Wang XY, Chen Y, Shah R, Yang HM, Cai Q.
Digestive system involvement of novel coronavírus infection: Prevention and control infection from a gastroenterology perspective. J Dig Dis. 2020 Apr;21(4):199-204.

15. An P, Ji M, Ren H, Su J, Ding NS, Kang J, Yin A, Zhou Q, Shen L, Zhao L, Jiang X, Xiao Y, Tan W, Lv X, Li J, Liu S, Zhou J, Chen H, Xu Y, Liu J, Chen M, Cao J, Zhou Z, Shen L, Tan S, Yu H, Dong W, Ding Y. Prevention of COVID-19 in patients with inflammatory bowel disease in Wuhan, China. Lancet Gastroenterol Hepatol. 2020;17.

16. Iacucci M, Cannatelli R, Labarile N, Mao R, Panaccione R, Danese S, Kochhar GS, Ghosh S, Shen B. Endoscopy in inflammatory bowel diseases during the COVID-19 pandemic and post-pandemic period. Lancet Gastroenterol Hepatol. 2020 Apr 16.

17. Cheung KS, Hung IF, Chan PP, Lung KC, Tso E, Liu R, Ng YY, Chu MY, Chung TW, Tam AR, Yip CC, Leung KH, YimFong Fung A, Zhang RR, Lin Y, Cheng HM, Zhang AJ, To KK, Chan KH, Yuen KY, Leung WK. Gastrointestinal Manifestations of SARS-CoV-2 Infection and Virus Load in Fecal Samples from the Hong Kong Cohort and Systematic Review and Meta-analysis. Gastroenterology. 2020;3.

18. Kotfis K, Skonieczna-Żydecka K. COVID-19: gastrointestinal symptoms and potential sources of 2019-nCoV transmission. Anaesthesiol Intensive Ther. 2020;23.

19. Wong SH, Lui RN, Sung JJ. Covid-19 and the digestive system. J Gastroenterol Hepatol. 2020;35(5):744-748.

20. Zhang JJ, Dong X, Cao YY, Yuan YD, Yang YB, Yan YQ, Akdis CA, Gao YD. Clinical characteristics of 140 patients infected with SARS-CoV-2 in Wuhan, China. Allergy. 2020;19.

21. Henry BM, de Oliveira MHS, Benoit J, Lippi G. Gastrointestinal symptoms associated with severity of coronavirus disease 2019 (COVID-19): a pooled analysis. Intern Emerg Med. 2020;17.

22. Zhou Z, Zhao N, Shu Y, Han S, Chen B, Shu X. Effect of Gastrointestinal Symptoms on Patients Infected With Coronavirus Disease 2019. Gastroenterology. 2020 Mar 19.

23. Lee IC, Huo TI, Huang YH. Gastrointestinal and Liver Manifestations in Patients with COVID-19. J Chin Med Assoc. 2020 Apr 1.

24. Chen Y, Chen L, Deng Q, Zhang G, Wu K, Ni L, Yang Y, Liu B, Wang W, Wei C, Yang J, Ye G, Cheng Z. The presence of SARS-CoV-2 RNA in the feces of COVID-19 patients. J Med Virol. 2020;3.

25. Tan YP, Tan BY, Pan J, Wu J, Zeng SZ, Wei HY. Epidemiologic and clinical characteristics of 10 children with coronavirus disease 2019 in Changsha, China. J Clin Virol. 2020;10;127:104353.

26. Xie M, Chen Q. Insight into 2019 novel coronavirus - An updated interim review and lessons from SARS-CoV and MERS-CoV. Int J Infect Dis. 2020 Apr 1;94:119-124.

27. Hajifathalian K, Mahadev S, Schwartz RE, Shah S, Sampath K, Schnoll-Sussman F, Brown RS Jr, Carr-Locke D, Cohen DE, Sharaiha RZ. SARS-COV-2 infection (coronavirus disease 2019) for the gastrointestinal consultant. World Gastroenterol. 2020;14;26(14):1546-1553.

28. Wang D, Hu B, Hu C, Zhu F, Liu X, Zhang J, Wang B, Xiang $\mathrm{H}$, Cheng Z, Xiong Y, Zhao Y, Li Y, Wang X, Peng Z. Clinical Characteristics of 138 Hospitalized Patients With 2019 Novel Coronavirus-Infected Pneumonia in Wuhan, China. JAMA. $2020 \mathrm{Feb} 7$.

29. Lu R, Zhao X, Li J, Niu P, Yang B, Wu H, Wang W, Song H, Huang B, Zhu N, Bi Y, Ma X, Zhan F, Wang L, Hu T, Zhou H, 
Hu Z, Zhou W, Zhao L, Chen J, Meng Y, Wang J, Lin Y, Yuan J, Xie Z, Ma J, Liu WJ, Wang D, Xu W, Holmes EC, Gao GF, Wu G, Chen W, Shi W, Tan W. Genomic characterisation and epidemiology of 2019 novel coronavirus: implications for virus origins and receptor binding. Lancet. 2020; 22;395(10224):565-574.
30. Han J, Wang Y, Zhu L, Cui Y, Li L, Zeng Z, Zhang S. Preventing the spread of COVID-19 in digestive endoscopy during the resuming period: meticulous execution of screening procedures. Gastrointest Endosc. 2020 Apr 5. 